\title{
Profiling substrate promiscuity of wild-type sugar kinases for multi- fluorinated monosaccharides
}

Tessa Keenan1,5, Fabio Parmeggiani2,5, Julien Malassis³, Clement Q. Fontenelle³, Jean-Baptiste Vendeville ${ }^{3}$, Wendy Offen¹, Peter Both ${ }^{2}$, Kun Huang2 2 Andrea Marchesi'2, Alex Heyam¹, Carl Young ${ }^{4}$, Simon, J. Charnock ${ }^{4}$, Gideon J. Davies ${ }^{1}$, Bruno Linclau ${ }^{3}$, Sabine L. Flitsch ${ }^{*}, 2$ Martin A. Fascione ${ }^{*}, 1,6$

${ }^{1}$ Department of Chemistry, University of York, Heslington, Y010 5DD, York, United Kingdom

${ }^{2}$ Manchester Institute of Biotechnology (MIB), School of Chemistry, University of Manchester, 131 Princess Street, M1 7DN, Manchester, United Kingdom

${ }^{3}$ School of Chemistry, University of Southampton, Highfield, S017 1BJ, Southampton, United Kingdom

4 Prozomix Limited, Haltwhistle Industrial Estate,, Haltwhistle, Northumberland, NE49 9HA, United Kingdom

${ }^{5}$ These authors contributed equally.

${ }^{6}$ Lead Contact

Correspondence:

martin.fascione@york.ac.uk

(M.A.F), $\quad$ sabine.flitsch@manchester.ac.uk

KEYWORDS: biocatalysis, kinases, sugar phosphates, fluorinated carbohydrates, oligosaccharides, glycobiology, enzyme discovery

SUMMARY: Fluorinated sugar-1-phosphates are of emerging importance as intermediates in the chemical and biocatalytic synthesis of modified oligosaccharides, as well as probes for chemical biology. Here we present a systematic study of the activity of a wide range of anomeric sugar kinases (galacto- and $\mathrm{N}$-acetylhexosamine kinases) against a panel of fluorinated monosaccharides, leading to the first examples of polyfluorinated substrates accepted by this class of enzymes. We have discovered four new $\mathrm{N}$-acetylhexosamine kinases with a different substrate scope, thus expanding the number of homologs available in this subclass of kinases. Lastly, we have solved the crystal structure of a galactokinase in complex with 2-deoxy-2fluoro galactose, giving insight into changes in the active site that may account for the specificity of the enzyme towards certain substrate analogues.

INTRODUCTION: Understanding the biological functions of complex oligosaccharides and the mechanisms that underpin carbohydrate recognition, synthesis and bioconversion, is an ever-expanding research area in glycobiology that has major implications in medicine and drug development. For these studies the supply of new substrates, probes and reference materials is often a major bottleneck, due to the high chemical complexity and cost of these target products. Although undoubtedly essential and powerful, the chemical synthesis of highly complex oligosaccharides such as blood group antigens, or sulfated glycosylaminoglycans, can be technically challenging and timeconsuming. ${ }^{1-4}$ As a complementary approach, chemo-enzymatic strategies, 5 in which chemical syntheses are used to generate natural and non-natural substrate analogues, before enzymatic functionalisation to produce key intermediates that enable access to a range of modified glycans, have also shown great promise. A representative example of this strategy is the synthesis of modified sugar-1-phosphates. Sugar-1-phosphates are important intermediates in sugar metabolism and utilization, ${ }^{6}$ being the first intermediate biosynthesised from the reducing monosaccharide, or the second intermediate biosynthesised from sugar-6-phosphates. Modified sugar-1-phosphates are commonly employed as basic building blocks towards the synthesis of diverse oligosaccharides, glycoproteins and glycosylated natural products, in their protected form as glycosyl donors for chemical syntheses, or unprotected as substrates in chemoenzymatic approaches.7,8 Fluorinated sugar-1-phosphates in particular, are important (Scheme 1) as it is well known that the presence of fluorine can influence the stereo-electronic properties of a molecule. ${ }^{9-11}$ Fluorinated sugar-1-phosphates are widely employed in chemo-enzymatic strategies for the synthesis of fluorinated glycans; For example, glycoside phosphorylases which use sugar-1-phosphates in catalysing the regio- and stereo-selective formation of glycosidic bonds, ${ }^{12}$ have been used for the chemo-enzymatic synthesis of fluorinated lacto- and galacto-n-bioses (galacto-n-biose/lacto-n-biose phosphorylase),13 enabling the synthesis of fluorinated T-antigen analogues. ${ }^{14}$ Monodeoxy-monofluorinated galactose-1-phosphates as substrates for uridyltransferases (GalUs) or UDP-sugar pyrophosphorylases (USPs), have been utilised in the synthesis of various fluorinated UDP-sugars, ${ }^{15}$ and larger glycans following their coupling via glycosyltransferases (GTs). ${ }^{16}$ Similarly, a UDP-6-deoxy-6fluoro-D-glucose was used as a donor for the synthesis of a glycosylated natural product, N-methylanthranilate. ${ }^{17}$ Furthermore, the peculiar stereo-electronic changes induced by the substitution of sugar ring hydroxyls for fluorine affects how these molecules interact with enzymes, ${ }^{18}$ making them extremely useful probes for structural and biochemical investigations.

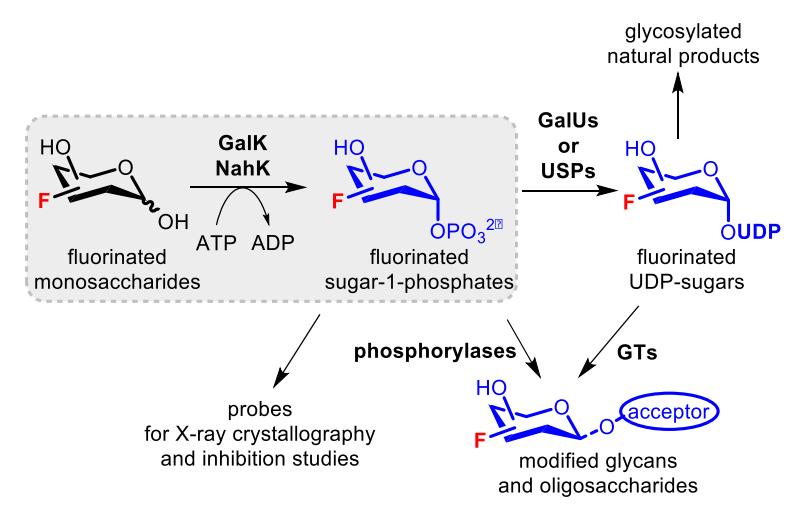

Scheme 1. Kinase-mediated synthesis of fluorosugar-1-phosphates and their applications. 
Table 1. Screening of fluorinated monosaccharides against GalKs and NahKsa.
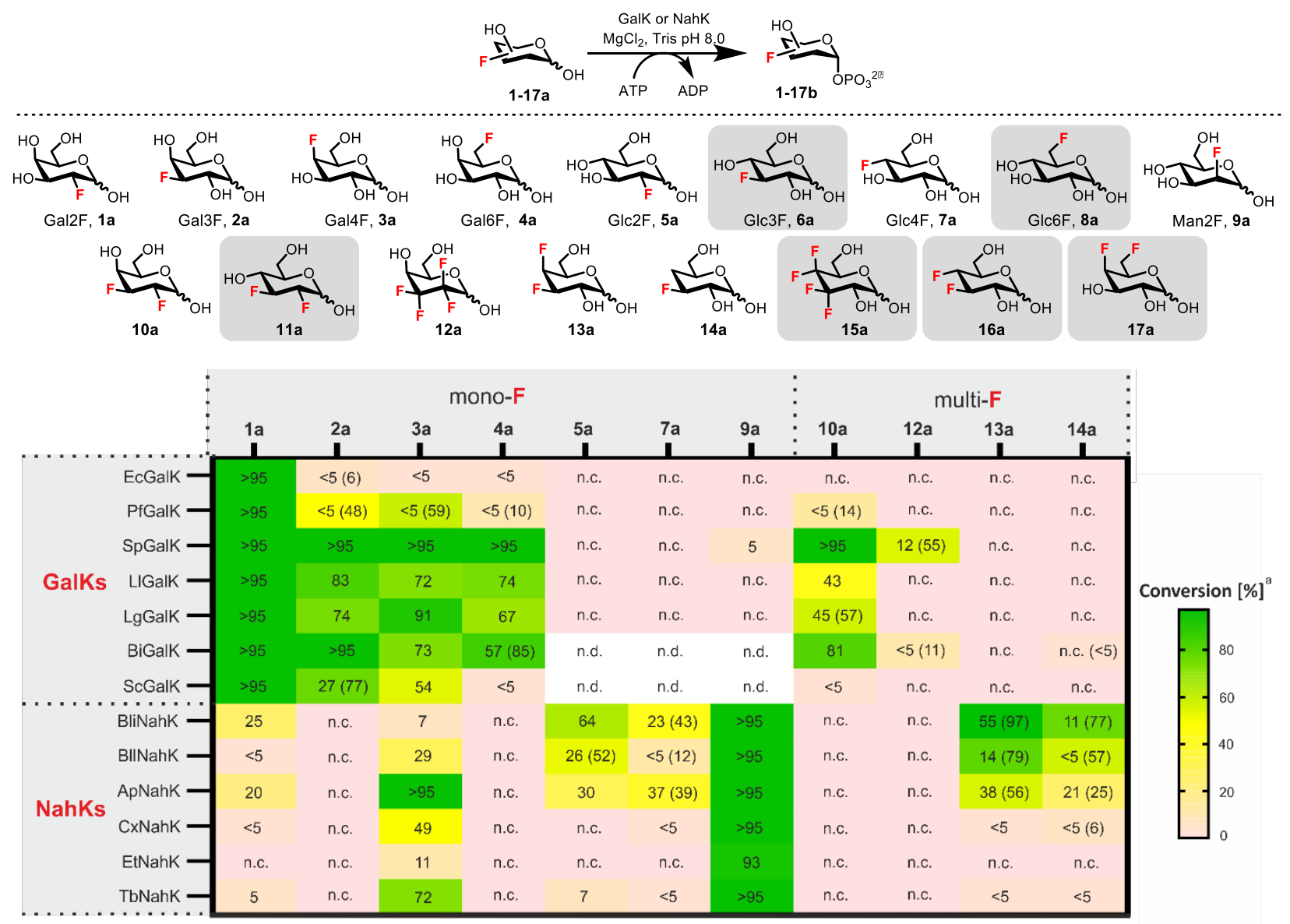

a Experimental conditions: $8 \mathrm{mM}$ substrate, $10 \mathrm{mM} \mathrm{ATP,} 5 \mathrm{mM} \mathrm{MgCl}_{2}, 0.12 \mathrm{mg} \mathrm{mL}^{-1} \mathrm{NahK}, 100 \mathrm{mM} \mathrm{Tris} \mathrm{pH} 8.0,37^{\circ} \mathrm{C}, 24 \mathrm{~h}^{\mathrm{b}}$ Determined by ${ }^{19} \mathrm{~F}$ NMR (n.c. $=$ no conversion, n.d. $=$ not determined). Optimised conversions shown in brackets. Limit of quantitation estimated at $5 \%$ conversion. Compounds highlighted in grey were not substrates for any kinases tested. New compounds 14a and 17a were synthesised as reported in the supporting information. Compounds 1a - 9a are commercially available. Known compounds 10a, 11a, 12a, 13a, 15a and 16a were synthesised, following protocols (see supporting information).

For example, UDP 2-deoxy-2-fluoro-galactose has been used to unpick mechanistic differences between $\alpha$ and $\beta$ galactosyltransferases (GalTs) through enzyme inhibition studies, ${ }^{19}$ and to investigate the catalytic mechanism of LgtC from Neisseria meningitidis through protein crystallography. The use of fluorinated carbohydrates in structural studies have also been key to identifying covalent reaction intermediates, as was demonstrated with the $\beta$-glucosidase $\mathrm{Cex},{ }^{20}$ and to gain insight into residues important in ligand recognition, as seen previously for mutases and synthases. 21,22 Fluorinated carbohydrates have proven to be useful as probes to study parasite adhesion in Toxoplasmosis. ${ }^{23}$ Additionally, they have been used as probes to map antibody sub-sites in anti-carbohydrate antibodies and in metabolic studies. 24,25

Two main classes of enzymes are responsible for the in vivo synthesis of sugar-1-phosphates from non-activated monosaccharides: galactokinases (GalKs) which catalyse the ATP dependant phosphorylation of $\alpha$-D-galactose (Gal) and $N$-acetylhexosamine kinases (NahKs, also known as HexNAcKs) which phosphorylate both $\mathrm{N}$-acetylglucosamine (GlcNAc) and $\mathrm{N}$-acetylgalactosamine (GalNAc). Many well characterised examples of GalKs have been reported,26-36 while only a few NahKs have been previously investigated.37,38 Both classes are highly promiscuous, exhibiting a broad tolerance to natural and derivatised monosaccharides. Despite the interest in fluorinated sugar-1-phosphates and the several published examples of GalKs and NahKs, few examples of kinase mediated fluorosugar phosphorylation have been demonstrated. All four monodeoxy-monofluoro galactoses
(2FGal 1a, 3FGal 2a, 4FGal 3a, 6FGal 4a) have long been recognised as substrates for yeast GalK, ${ }^{39}$ and investigated subsequently as model substrates with EcGalK ${ }^{15,17}$ and LgGalK. ${ }^{32}$ Some groups have also reported the conversion of geminally fluorinated monosaccharide derivatives, such as 6,6-difluoro-D-fucose (6-deoxy-6,6-difluoro-Dgalactose) 40,41 and N-trifluoroacetyl-D-glucosamine/-Dgalactosamine. ${ }^{38}$ However, the tolerance of anomeric kinases to vicinal dideoxy-difluorinated substrates, or monosaccharides with a higher degree of fluorination, is currently unexplored. This class of sugars is of interest in the study of protein-carbohydrate interactions, due to their polar hydrophobicity which can increase the affinity of these compounds for proteins. ${ }^{18,22,42-46}$ Here, we describe a systematic approach in which fluorosugar reactivity of diverse anomeric kinases was explored, leading to examples of mono-, di- and tetrafluorinated substrates that are phosphorylated by these enzymes. We also describe the 3D-structure of BiGalK and showcase, through ligand complexes with $2 \mathrm{FGal}$ and Gal, subtle changes in the active site which can occur when a sugar ring-hydroxyl is replaced with fluorine.

RESULTS AND DISCUSSION: A panel of seven published GalKs (EcGalK ${ }^{29}$, PfGalK,35 SpGalK,30 LlGalK,36 LgGalK,32 BiGalK ${ }^{31}$ and ScGalK $^{34}$ ) and two published NahKs (BliNahK ${ }^{38}$ and BllNahK ${ }^{38}$ ) was chosen. Due to the lower diversity available in the latter family, four putative NahK homologs (49-54\% sequence identity) from bacterial species (Supporting information) were also selected and included in the panel: ApNahK (WP_091282512), CxNahK (WP_045094128), EtNahK (ODM08532) and TbNahK (WP_062612735). The kinase panel was investigated for the conversion of a series of deoxyfluorinated 
galactoses, glucoses and a mannose derivative. While some examples of kinase mediated phosphorylation of monofluorinated galactoses have been reported previously15,17,32,39, we rationalised that the inclusion of these compounds in our extensive screen could help to identify kinases with higher activity against these, as well as with a broader substrate scope. Kinase mediated conversion of the substrates to the sugar-1-phosphate was determined by ${ }^{19} \mathrm{~F}$ NMR (Table 1, Table S1) by relative integration of the corresponding resonances which were typically baseline-separated. Phosphorylation at the anomeric position of the monosaccharides was evident, as shown by the formation of only a single new species in the ${ }^{19} \mathrm{~F}$ NMR, in comparison to two species that were present in the reference spectrum of the monosaccharide, where the anomeric centre is in the hemiacetal form. Formation of the sugar-1-phosphate was further validated by HRMS analysis (Supporting information).

As expected, the GalKs demonstrated a strict preference for deoxyfluorinated galactoses. Deoxyfluorination at the 2-position was the most tolerated, as demonstrated by the near full conversion of 2FGal (1a) by all of the GalKs. All GalKs displayed some activity towards 3FGal (2a), 4FGal (3a) and 6FGal (4a), however there were clearly differences in the activity profiles towards these substrates, between the different enzymes. For example, BiGalK catalysed nearly full conversion of $3 \mathrm{FGal}$ but moderate conversions of $4 \mathrm{FGal}$ and $6 \mathrm{FGal}$. In contrast, ScGalK catalysed moderate conversion of $4 \mathrm{FGal}$ but lowlevel conversions of 3FGal and 6FGal. Overall, SpGalK catalysed the highest conversions of all deoxyfluorinated galactoses (>95\%), as well as the conversion of 2 FMan (9a) at low levels, further indicating its promiscuity.

The NahKs displayed a broader fluorinated substrate scope, demonstrating the conversion of some deoxyfluorinated galactoses, glucoses and the mannose derivative. Most NahKs displayed moderate activity towards 2- and 4- deoxyfluorinated analogues, while displaying little or no activity towards their 3- and 6- deoxyfluorinated counterparts. As expected, most of the NahKs phosphorylated both galacto- and gluco- configured substrates. This could be explained by a lack of hydrogen bonding interactions made between the enzyme active site residues and the $4-\mathrm{OH}$, as is shown in the crystal structure of BliNahK in complex with GlcNAc ${ }^{47}$ (Figure S1). All NahKs catalysed the highest conversions of 2FMan (9a). In previous studies by Chen, Wang and colleagues, 38 BllNahK and BliNahK were shown to be highly promiscuous enzymes, not only demonstrating the efficient conversion of GalNAc and GlcNAc and derivatives, but also mannose and its C-2, C4 and C- 6 derivatives, albeit with lower specific activities. They were also shown to convert glucose and galactose, although to a lesser extent than mannose. Their findings are consistent with our NahK screening results, which suggest that 2FMan is a better substrate for NahKs than any of the galcto- or gluco- configured substrates.

The panel of kinases was then investigated for activity towards vicinal dideoxygenated monosaccharides having di- and tetrafluorinated motifs (Table 1, Table S2). Interestingly, most of the GalKs phosphorylated 2,3-dideoxy-2,3-difluoro galactose (10a) (Figure S4) but not 3,4-dideoxy-3,4-difluorogalactose (13a) or 4,6-dideoxy-4,6difluoro galactose (17a), despite being able to convert monofluorinated monosaccharides 3FGal, 4FGal and 6FGal. Notably, SpGalK and BiGalK also converted 2,3-dideoxy-2,2,3,3-tetrafluoro-Dthreo-hexopyranose (12a) but not 3,4-dideoxy-3,3,4,4-tetrafluoro-Derythro-hexopyranose (15a). Although only trace amounts of product was observed under normal screening conditions, reaction optimisation by increasing the concentration of SpGalK and the reaction time, improved the conversion to $55 \%$ (Figure 1). The chemically synthesised sugar phosphate, 2,3-dideoxy-2,2,3,3tetrafluoro-D-threo-hexopyranose phosphate $(\mathbf{1 2 b})^{46}$ was used as a ${ }^{19} \mathrm{~F}$ NMR standard to validate the presence of the correct product. The findings that GalKs could only catalyse the conversion of vicinal di- and tetra-fluorinated galactoses modified at the 2- and 3- positions, further support observations made in the deoxyfluorinated monosaccharide screening, which highlighted the high activity of GalKs (particularly SpGalK and BiGalK) towards 2- and 3-deoxyfluorinated galactose derivatives. On the other hand, the GalKs did not show turnover of the gluco-configured substrates 11a and 16a or the vicinal 3,4-dideoxy-3fluoro sugar (14a), further emphasising the importance of galactoconfiguration at the 4-position in determining GalK substrate preference. In contrast to the GalKs, the NahKs were moderately active towards 3,4-dideoxy-3,4-difluorogalactose (13a) and the 3,4-dideoxy3 -fluoro sugar (14a) and conversions of up to $97 \%$ and $77 \%$ could be obtained for 13a and 14a respectively (Table 1), but displayed no activity towards 3,4-dideoxy-3,4-difluoroglucose (16a). Despite increasing the enzyme loading and reaction times, NahKs failed to reveal any activity towards analogues 10a, 11a, 12a, 15a or 17a. Considering that a single fluorine on a monosaccharide substrate can lead to reduced enzyme catalytic efficiency, ${ }^{48}$ remarkably within this panel of 8 heavily fluorinated monosaccharides, 4 of them could be effectively phosphorylated ( $>50 \%$ conversion) by at least one of the kinases investigated.
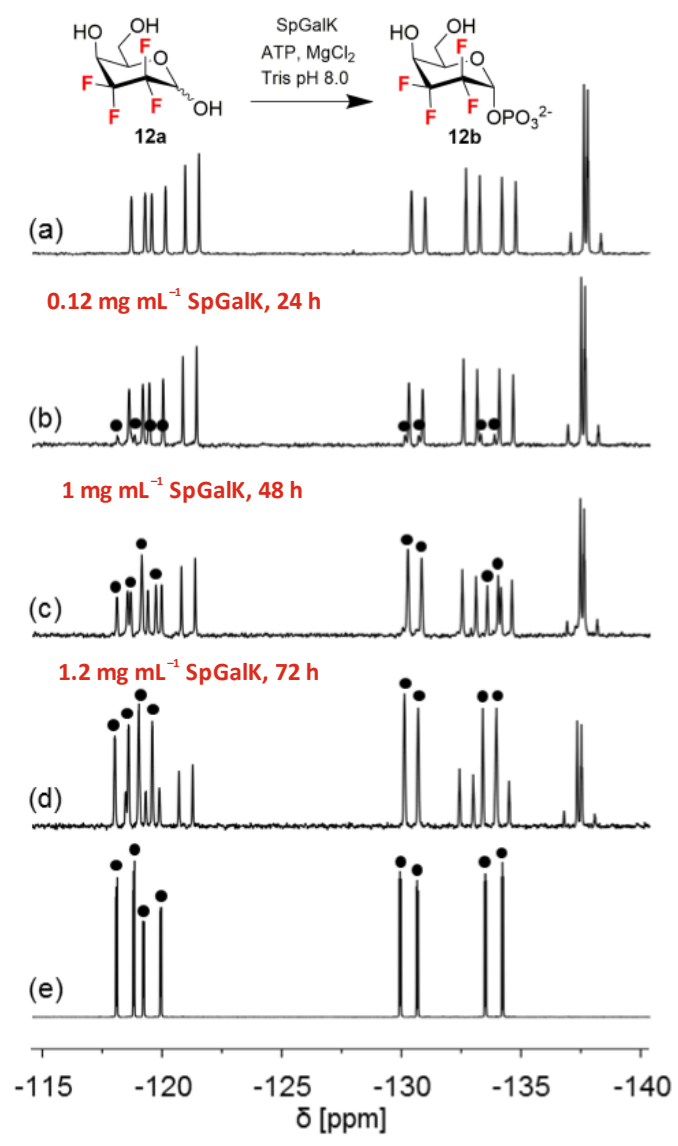

Figure 1. ${ }^{19} \mathrm{~F}$ NMR analysis of SpGalK mediated phosphorylation of substrate 12a. Peaks corresponding to product 12b are marked ( ). (a) Reference spectrum of 12a; (b) Reaction under screening conditions; (c-d) Reactions with increased loading and reaction times; (e) reference spectrum of $\mathbf{1 2 b . 4 6}$

The preference of GalKs for galacto-configured substrates fluorinated at the 2-position, emphasised by the preference of difluorinated analogue 10a over analogues 13a and 17a, prompted us to investigate these interactions at the molecular level. Using BiGalK as a model example, the crystal structures of BiGalK in complex with 2FGal 1a (PDB: 6TEQ), Gal (PDB: 6TER) and ADP (PDB: 6TEP) were solved (Table S3). Overall, the secondary structure architectures of BiGalK 
were nearly indistinguishable between the three ligand-bound complexes (Figure S2a). The position of the bound ADP was as expected (Figure S2b), when compared to the nucleotide binding sites in the structures of $\mathrm{LlGalK}^{49}$ and PfGalK. ${ }^{35}$ In the sugar-bound complexes 2FGal (1a) and Gal are present as a mixture of the alpha and beta forms. In the Gal-bound structure, the ligand is positioned through a network of hydrogen bonding interactions (Figure 2a).
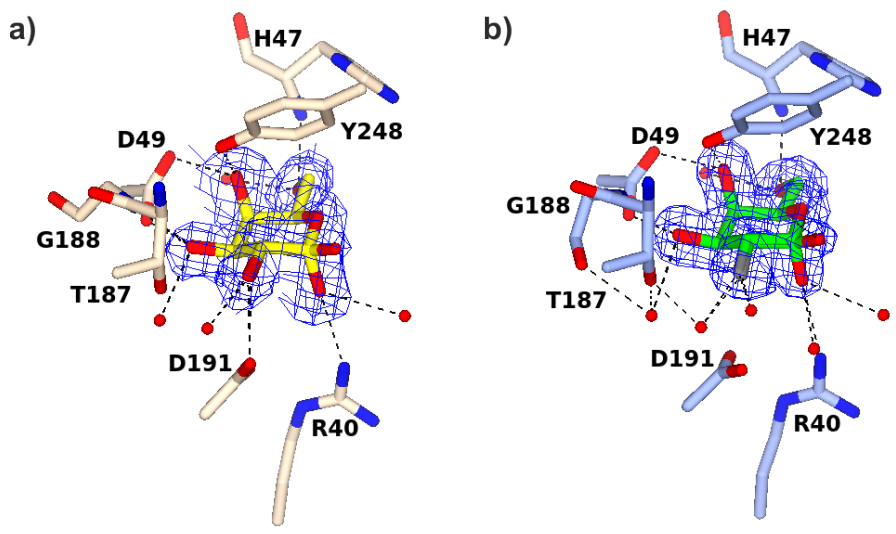

Figure 2. BiGalK active site residues interacting with Gal (a) and 2FGal (b). Electron density maps are REFMAC maximum-likelihood/ $\sigma \mathrm{A}$ weighted 2Fo-Fc maps contoured at an r.m.s.d. level of $1.0 \sigma$ for both ( 0.31 and 0.36 electrons $/ \AA^{3}$ for (a) and (b), respectively). Alpha and beta anomers are modelled at 0.9/0.1 occupancy in (a) and 0.6/0.4 occupancy in (b), respectively.

The active site residues that interact directly with Gal (R40, H47, D49, G188, D191, Y248) are conserved in all bacterial GalKs investigated here (Figure S3). As expected for the alpha-configured sugar, the 1-OH is positioned within hydrogen bonding distance of R40 and close to D191 (within 3.2-3.6 ̊̊). The corresponding residues in L. lactis GalK (R37, D186) were previously shown to have a role in catalysis by deprotonating the 1-OH of Gal. ${ }^{49}$ The 2-OH forms hydrogen bonding interactions with the side chain of D191 and some water molecules, while the 3-OH hydrogen bonds to OD2 D49, N G188 and a water molecule. D49, which forms hydrogen bonds with both the 3-OH and 4-OH, appears to be important for substrate positioning. This potentially accounts for the absence of GalK activity towards 13a, as fluorines at the 3- and 4-positions could not act as hydrogen bond donors. Whilst the sugar molecule is relatively accessible near the 2$\mathrm{OH}$ where there is a network of hydrogen-bonded water molecules, the active site residues interact more closely around the 4- $\mathrm{OH}$ and 6-OH.

In the 2FGal-bound structure, the 2-fluorine can only serve as a hydrogen bond acceptor and therefore cannot form a hydrogen bond with the side chain of D191 (Figure 2b). There are one or two additional water molecules modelled in the region close to, and forming hydrogen bonds with the 2-fluorine in the 2FGal structure molecules, compared to those observed for the 2-OH group in the Gal structure. These extra water molecules may help to compensate for the loss of interactions with D191. The carbonyl group of G188 is flipped, allowing it to form a new hydrogen bond with the water molecule that interacts with the 3-0H. OG1 T187 appears to stabilise the water molecules which interact with the 2-fluorine and the 3-OH. These subtle variations in the ligand-water hydrogen bonding network may explain why fluorination at the 2- and 3-positions is well tolerated by the enzyme. However, the kinetic parameters of BiGalK towards Gal $\left(\mathrm{K}_{\mathrm{m}}=1.029 \mathrm{mM}, \mathrm{K}_{\text {cat }} / \mathrm{K}_{\mathrm{m}}=132.32 \mathrm{~s}^{-1} \mathrm{mM}^{-1}\right)$ and $2 \mathrm{FGal}\left(\mathrm{K}_{\mathrm{m}}=4.742 \mathrm{mM}\right.$, $\mathrm{K}_{\mathrm{cat}} / \mathrm{K}_{\mathrm{m}}=13.5 \mathrm{~s}^{-1} \mathrm{mM}^{-1}$ ) show that even a single fluorine at the 2- position results in a near 10 -fold decrease in the catalytic efficiency of the enzyme.

One could envisage that the restricted space around the 4-OH and 6$\mathrm{OH}$ prohibits access for water molecules which could form compensatory hydrogen bonds when these groups are substituted with fluorine, as in 17a. This observation is reflected in the decreasing conversions when using BiGalK as the number of fluorines around the substrates ring increases, when comparing monofluorinated Gal2F 1a ( $>95 \%$ conversion), to 2,3-dideoxy-2,3-difluoro galactose 10a $(81 \%)$, 2,3-dideoxy-2,2,3,3-tetrafluoro-D-threo-hexopyranose 12a $\quad(<5 \%)$. Indicating that if a fluorine is present at position 2 in the ring, fluorination at other positions may be tolerated, but with an increasing penalty to reactivity. Overall, the structure showcases several residues that may be good targets for mutagenesis to expand BiGalK specifity towards fluorinated substrates. Residues H47 and Y248 that interact closely with the 4-OH and 6-OH would be good targets to explore for expanding BiGalK activity towards 17a. When considering substrates with fluorination at the 3- and 4-positions, Y248, G188 and T187 could be targeted. Considering the apparent role of D49 for achieving correct substrate positioning, it is likely that mutations to this residue may completely eliminate enzyme activity.

CONCLUSIONS: In summary, we have demonstrated that wild-type anomeric sugar kinases can be exploited to access fluorinated monosaccharide-1-phosphates. We have systematically profiled kinase activity towards fluorinated analogues, demonstrating the first examples of polyfluorinated substrates accepted by this enzyme class. Our screen consisted of a large and diverse panel of kinases, highlighting that the selection of homologs with low sequence similarity can facilitate the identification of novel substrate specificities. We have shown that GalKs show a strong preference for galacto-configuration and a 4-OH. Deoxyfluorination at the 2-position was most tolerated by the GalKs, and this was further reinforced by observation that only the vicinal dideoxy-difluorinated galactose modified at the 2- and 3-positions (10a) was turned over by the enzymes. Furthermore, 2,3-dideoxy-2,2,3,3-tetrafluoro-D-threohexopyranose (12a) was converted by SpGalK and BiGalK, with a conversion of $55 \%$ achieved under optimised reaction conditions. In contrast, NahKs displayed a broader fluorosugar substrate range than the GalKs, catalysing the highest conversions of mannose derivative 2FMan (9a), but accepting both galacto- and gluco-configured substrates. They displayed a strict preference towards 2- and 4deoxyfluorinated analogues and catalysed the conversion of vicinal dideoxy-difluorinated sugars, 3,4-dideoxy-3,4-difluorogalactose (13a) and the 3,4-dideoxy-3-fluoro sugar (14a). Overall, we have demonstrated the effective phosphorylation of 11 different fluorinated monosaccharides and through optimised reaction conditions, obtained conversions of $>90 \%$ for 7 of these, setting the scene for further optimisation by rational design and/or random mutagenesis. Through the structural characterisation of a GalK in complex with $2 \mathrm{FGal}$, we have gained valuable insight into subtle changes in the active site which can occur when a sugar ring-hydroxyl is replaced with fluorine. These results pave the way to the one-step synthesis of useful intermediates for enzymatic glycosylations, to be explored through the synthesis of UDP-sugars (GalUs/USPs) and their coupling via GTs, or directly via glycosyl phosphorylases. They may also serve as useful probes for chemical biology, in structural studies to inform proteinligand interactions. 


\section{Supporting information}

Experimental details and supporting data (PDF)

\section{ORCID}

Tessa Keenan: 0000-0003-2466-6918

Fabio Parmeggiani: 0000-0001-5861-9269

Clement Q. Fontenelle: 0000-0002-1630-3407

Jean-Baptiste Vendeville: 0000-0002-5790-6968

Wendy Offen: 0000-0002-2758-4531

Kun Huang: 0000-0003-0269-2963

Andrea Marchesi: 0000-0002-1560-5921

Gideon Davies: 0000-0002-7343-776X

Bruno Linclau: 0000-0001-8762-0170

Sabine Flitsch: 0000-0003-3974-646X

Martin Fascione: 0000-0002-0066-4419

\section{AUTHOR CONTRIBUTIONS}

F.P., T.K., K.H. and A.M. performed the enzymatic transformations; J.M., C.Q.F. and J.B.V. performed chemical synthesis and characterisation; T.K. and W.O. performed crystallography; P.B., C.Y. and T.K. performed sequence identification, cloning and protein production; A.H. developed ${ }^{19} \mathrm{~F}$ NMR methodology; M.A.F., S.L.F., B.L., G.J.D., S.J.C. supervised the project; M.A.F., S.L.F. and B.L. designed the study; T.K. and F.P. wrote the paper, and all authors commented on the paper.

\section{NOTES}

The authors declare no competing financial interests: Prozomix is a commercial enzyme producer.

\section{ACKNOWLEDGEMENTS}

This project has been funded by the Industrial Biotechnology Catalyst (Innovate UK, BBSRC, EPSRC) to support the translation, development and commercialisation of innovative Industrial Biotechnology processes: $\quad \mathrm{BB} / \mathrm{M} 028836 / 1$; $\mathrm{BB} / \mathrm{L} 013762 / 1$, BB/M027791/1; BB/M02903411; BB/M028836/1; BB/M028941/1; BB/M02847X/1, EP/K039466/1 and ERC (788231-ProgrES-ERC-2017-ADG). Dr. Matt Cliff and Dr. Reynard Spiess (University of Manchester) are acknowledged for their support in NMR and HRMS analysis, respectively. The York Centre of Excellence in Mass Spectrometry was created thanks to a major capital investment through Science City York, supported by Yorkshire Forward with funds from the Northern Way Initiative, and subsequent support from EPSRC (EP/K039660/1; EP/M028127/1).

\section{REFERENCES}

1. Maza, S. et al. Synthesis of amine-functionalized heparin oligosaccharides for the investigation of carbohydrateprotein interactions in microtiter plates. Org. Biomol. Chem. 10, 2146-2163 (2012).

2. Xia, J. et al. Synthesis of fluorinated mucin core 2 branched oligosaccharides with the potential of novel substrates and enzyme inhibitors for glycosyltransferases and sulfotransferases. J. Org. Chem. 71, 3696-3706 (2006).

3. Hanashima, S., Castagner, B., Esposito, D., Nokami, T. \& Seeberger, P.H. Synthesis of a sialic acid alpha(2-3) galactose building block and its use in a linear synthesis of sialyl Lewis X. Org. Lett. 9, 1777-9 (2007).

4. Tiruchinapally, G., Yin, Z., El-Dakdouki, M., Wang, Z. \& Huang, $\mathrm{X}$. Divergent heparin oligosaccharide synthesis with preinstalled sulfate esters. Chem. Eur. J. 17, 10106-10112 (2011).

5. Muthana, S., Cao, H. \& Chen, X. Recent progress in chemical and chemoenzymatic synthesis of carbohydrates. Curr. Opin. Chem. Biol. 13, 573-581 (2009).

6. Varki, A. et al. Essentials of Glycobiology [Internet]. 3rd edition. Cold Spring Harbor (NY): Cold Spring Harbor Laboratory Press (2015-2017).

7. Plante, O.J., Palmacci, E.R., Andrade, R.B. \& Seeberger, P.H. Oligosaccharide synthesis with glycosyl phosphate and dithiophosphate triesters as glycosylating agents. J. Am. Chem. Soc. 123, 9545-9554 (2001).

8. Fu, X. et al. Antibiotic optimization via in vitro glycorandomization. Nat. Biotechnol. 21, 1467 (2003).

9. Gillis, E.P., Eastman, K.J., Hill, M.D., Donnelly, D.J. \& Meanwell, N.A. Applications of fluorine in medicinal chemistry. J. Med. Chem. 58, 8315-8359 (2015).

10. Wang, J. et al. Fluorine in pharmaceutical industry: fluorinecontaining drugs introduced to the market in the last decade (2001-2011). Chem. Rev. 114, 2432-2506 (2013).

11. O'Hagan, D. Understanding organofluorine chemistry. An introduction to the C-F bond. Chem. Soc. Rev. 37, 308-319 (2008).

12. O'Neill, E.C. \& Field, R.A. Enzymatic synthesis using glycoside phosphorylases. Carbohydrate research 403, 23-37 (2015).

13. Yu, H. et al. Highly efficient chemoenzymatic synthesis of $\beta 1-$ 3-linked galactosides. Chem. Commun. 46, 7507-7509 (2010).

14. Yan, J., Chen, X., Wang, F. \& Cao, H. Chemoenzymatic synthesis of mono-and di-fluorinated Thomsen-Friedenreich (T) antigens and their sialylated derivatives. Org. Biomol. Chem. 11, 842-848 (2013).

15. Errey, J.C., Mukhopadhyay, B., Kartha, K.R. \& Field, R.A. Flexible enzymatic and chemo-enzymatic approaches to a broad range of uridine-diphospho-sugars. Chem. Commun., 2706-2707 (2004).

16. Kodama, H., Kajihara, Y., Endo, T. \& Hashimoto, H. Synthesis of UDP-6-deoxy-and-6-fluoro-D-galactoses and their enzymatic glycosyl transfer to mono-and biantennary carbohydrate chains. Tetrahedron Lett. 34, 6419-6422 (1993).

17. Caputi, L. et al. A one-pot enzymatic approach to the Ofluoroglucoside of N-methylanthranilate. Bioorg. Med. Chem 21, 4762-4767 (2013).

18. Ioannou, A. et al. Heavily fluorinated carbohydrates as enzyme substrates: oxidation of tetrafluorinated galactose by galactose oxidase. Chem. Commun. 47, 11228-11230 (2011).

19. Burkart, M.D. et al. Chemo-enzymatic synthesis of fluorinated sugar nucleotide: useful mechanistic probes for glycosyltransferases. Bioorg. Med. Chem. 8, 1937-1946 (2000).

20. White, A., Tull, D., Johns, K., Withers, S.G. \& Rose, D.R. Crystallographic observation of a covalent catalytic intermediate in a $\beta$-glycosidase. Nat. Struct. Biol. 3, 149-154 (1996).

21. Gibson, R.P., Tarling, C.A., Roberts, S., Withers, S.G. \& Davies, G.J. The donor subsite of trehalose-6-phosphate synthase binary complexes with UDP-glucose and UDP-2-deoxy-2fluoro-glucose at $2 \AA$ Å resolution. J. Biol. Chem. 279, 1950 1955 (2004).

22. Van Straaten, K.E. et al. Structural basis of ligand binding to UDP-galactopyranose mutase from Mycobacterium tuberculosis using substrate and tetrafluorinated substrate analogues. J. Am. Chem. Soc. 137, 1230-1244 (2015).

23. Allman, S.A. et al. Potent Fluoro-oligosaccharide Probes of Adhesion in Toxoplasmosis. ChemBioChem 10, 2522-2529 (2009). 
24. Schmidt, M.F., Biely, P., Kratky, Z. \& Schwartz, R.T.

Metabolism of 2-Deoxy-2-fluoro-d-[3H] glucose and 2-Deoxy2-fluoro-d-[3H] mannose in Yeast and Chick-Embryo Cells.

Eur. J. Biochem. 87, 55-68 (1978).

25. Glaudemans, C.P. Mapping of subsites of monoclonal, anticarbohydrate antibodies using deoxy and deoxyfluoro sugars. Chem. Rev. 91, 25-33 (1991).

26. Chu, X., Li, N., Liu, X. \& Li, D. Functional studies of rat galactokinase. J. Biotechnol. 141, 142-146 (2009).

27. Dey, P.M. Galactokinase of Vicia faba seeds. Eur. J. Biochem. 136, 155-159 (1983).

28. Schell, M. \& Wilson, D. Purification and properties of galactokinase from Saccharomyces cerevisiae. J. Biol. Chem. 252, 1162-1166 (1977).

29. Yang, J. et al. Studies on the substrate specificity of Escherichia coli galactokinase. Org. Lett. 5, 2223-2226 (2003).

30. Chen, M. et al. Wide sugar substrate specificity of galactokinase from Streptococcus pneumoniae TIGR4. Carbohydr. Res. 346, 2421-2425 (2011).

31. Li, L. et al. A highly efficient galactokinase from Bifidobacterium infantis with broad substrate specificity. Carbohydr. Res. 355, 35-39 (2012).

32. Huang, K. et al. Characterisation of a Bacterial Galactokinase with High Activity and Broad Substrate Tolerance for Chemoenzymatic Synthesis of 6-Aminogalactose-1Phosphate and Analogues. ChemBioChem 19, 388-394 (2018).

33. Li, S.P. et al. Characterization of Meiothermus taiwanensis Galactokinase and its use in the one-pot enzymatic synthesis of Uridine Diphosphate-Galactose and the chemoenzymatic synthesis of the carbohydrate antigen stage specific embryonic antigen-3. Adv. Synth. Catal. 356, 3199-3213 (2014).

34. Keenan, T. et al. The characterisation of a galactokinase from Streptomyces coelicolor. Carbohydr. Res. 472, 132-137 (2019).

35. Hartley, A. et al. Substrate specificity and mechanism from the structure of Pyrococcus furiosus galactokinase. J. Mol. Biol. 337, 387-398 (2004).

36. Grossiord, B.P., Luesink, E.J., Vaughan, E.E., Arnaud, A. \& de Vos, W.M. Characterization, expression, and mutation of the Lactococcus lactis galPMKTE genes, involved in galactose utilization via the Leloir pathway. J. Bacteriol. 185, 870-878 (2003).

37. Cai, L. et al. Substrate specificity of $\mathrm{N}$-acetylhexosamine kinase towards $\mathrm{N}$-acetylgalactosamine derivatives. Bioorg. Med. Chem. Lett. 19, 5433-5435 (2009).

$38 . \quad \mathrm{Li}, \mathrm{Y}$. et al. Substrate promiscuity of $\mathrm{N}$-acetylhexosamine 1kinases. Molecules 16, 6396-6407 (2011).

39. Thomas, P., Bessell, E.M. \& Westwood, J.H. The use of deoxyfluoro-D-galactopyranoses in a study of yeast galactokinase specificity. Biochem. J. 139, 661-664 (1974).

40. Yang, J., Liu, L. \& Thorson, J.S. Structure-based enhancement of the first anomeric glucokinase. ChemBioChem 5, 992-996 (2004).

41. Yang, J., Fu, X., Liao, J., Liu, L. \& Thorson, J.S. Structure-based engineering of $E$. coli galactokinase as a first step toward in vivo glycorandomization. Chem. Biol. 12, 657-664 (2005).

42. Biffinger, J.C., Kim, H.W. \& DiMagno, S.G. The polar hydrophobicity of fluorinated compounds. ChemBioChem $\mathbf{5}$, 622-627 (2004).

43. Kim, H.W., Rossi, P., Shoemaker, R.K. \& DiMagno, S.G. Structure and transport properties of a novel, heavily fluorinated carbohydrate analogue. J. Am. Chem. Soc. 120, 9082-9083 (1998).

44. Bresciani, S., Lebl, T., Slawin, A.M. \& O'Hagan, D.

Fluorosugars: synthesis of the 2, 3, 4-trideoxy-2, 3, 4-trifluoro hexose analogues of D-glucose and D-altrose and assessment of their erythrocyte transmembrane transport. Chem.

Commun. 46, 5434-5436 (2010).
45. Denavit, V. et al. Stereoselective Synthesis of Fluorinated Galactopyranosides as Potential Molecular Probes for Galactophilic Proteins: Assessment of Monofluorogalactoside-LecA Interactions. Chem. Eur. J. 25, 4478-4490 (2019).

46. N'Go, I. et al. Tetrafluorination of sugars as strategy for enhancing protein-carbohydrate affinity: Application to UDPGalp mutase inhibition. Chem. Eur. J. 20, 106-112 (2014).

47. Wang, K.-C. et al. Insights into the binding specificity and catalytic mechanism of $\mathrm{N}$-acetylhexosamine 1-phosphate kinases through multiple reaction complexes. Acta Cryst. D. 70, 1401-1410 (2014).

48. Council, C.E. et al. Enzymatic glycosylation involving fluorinated carbohydrates. Organic \& Biomolecular Chemistry (2020).

49. Thoden, J.B. \& Holden, H.M. Molecular structure of galactokinase. J. Biol. Chem. 278, 33305-33311 (2003).

TOC graphic

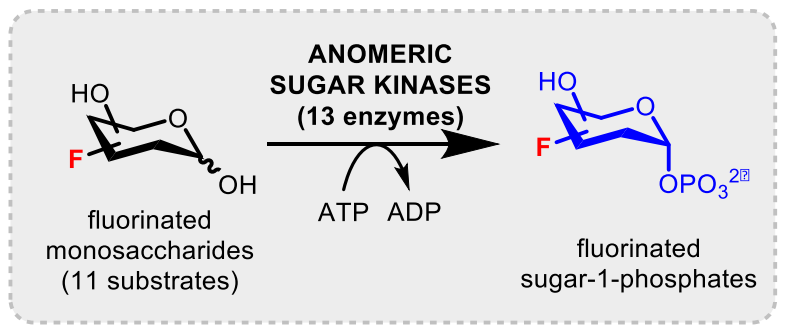

